\title{
The Magnetic Reorientation in Ferrimagnetic Systems
}

\author{
V. ILKOVIC̆*
}

Bogoliubov Laboratory of Theoretical Physics, JINR, 141980, Dubna, Moscow region, Russia

Permanent address: Institute of Experimental Physics, Slovak Academy of Sciences, Watsonova 47,04001 Košice, Slovakia

\begin{abstract}
The Green function theory is used to study the phenomenon of magnetic reorientation in the ferrimagnetic system. The two-sublattice ferrimagnetic system, with different spins and the intra-sublattice ferromagnetic exchange interactions, is considered. The magnon fluctuations supress in different way the magnetic orders of the sublattices and one obtains two magnetic orders. Each of these orders has different reorientation temperatures.
\end{abstract}

DOI: $10.12693 /$ APhysPolA.126.34

PACS: 75.10.Jm, 75.30.Ds, 75.30.Kz, 75.70.Ak

\section{Introduction and fundamental equations}

The phenomenon of the magnetic reorientation can be qualitatively understood by consideration of the competing forces that favour different directions of the magnetization such as easy-axis single-ion anisotropy and dipolar interaction. In addition, the magnetic reorientation can be induced by applying a magnetic field [1].

We investigate the magnetic reorientation within the Heisenberg model for the ferrimagnetic system in the approximation of two-sublattices with spins $S_{A}$ and $S_{B}$ at the sublattice $A$ and $B$ sites, respectively. The Hamiltonian can be written as

$$
\begin{aligned}
H & =\frac{1}{2} J \sum_{\langle i A, j B\rangle}\left(S_{i A}^{-} S_{j B}^{+}+S_{i A}^{z} S_{j B}^{z}\right)-\frac{1}{2} \sum_{\alpha=A, B} \sum_{\langle i, j\rangle} J_{\alpha}\left(S_{i \alpha}^{-} S_{j \alpha}^{+}+S_{i \alpha}^{z} S_{j \alpha}^{z}\right)+\frac{1}{2} D \sum_{\langle i A, j B\rangle} S_{i A}^{z} S_{j B}^{z} \\
& -\sum_{\alpha=A, B} \sum_{i} K_{2}\left(S_{i \alpha}^{z}\right)^{2}-h^{x} \sum_{\alpha=A, B} \sum_{i} S_{i \alpha}^{x} .
\end{aligned}
$$

The first term is the operator of the inter-sublattice antiferromagnetic exchange interaction energy $(J>0)$; the second term corresponds to two operators of the intra-sublattice ferromagnetic exchange interaction energy. The parameter $D>0$ denotes the inter-sublattice exchange anisotropy. The fourth term represents the single-ion anisotropy. The transverse magnetic field $h^{x}$ is shorthand for $g \mu_{B} H$.

The applied Green function method in this work was described in detail elsewhere [2-5]. The theory is based on a transformation of the fixed coordinate system $(x, y, z)$ into a local coordinate system $(X, Y, Z)$. The $Z$ axis is set to be parallel to the magnetization direction. A remarkable result of this theory is that the effective field aligned parallel to the $Z$ axis can be written as a sum of the external magnetic field and an anisotropy field:

$$
\left.h=h^{x} \sin \theta+2 K_{2}\left\langle S_{\alpha}^{Z}\right\rangle\left(\cos ^{2} \theta_{\alpha}-\left(\sin ^{2} \theta_{\alpha}\right) / 2\right]\right) Q^{\left(S_{\alpha}\right)},
$$

with $Q_{\alpha}^{\left(S_{\alpha}\right)}=1-\left[S_{\alpha}\left(S_{\alpha}+1\right)-\left\langle\left(S_{\alpha}^{Z}\right)^{2}\right\rangle\right] / 2 S_{\alpha}^{2},(\alpha=A, B)$. Angle $\theta_{\alpha}$ denotes the orientation angle of the magnetization. We introduce the Fourier transform Green functions $(\mathrm{GF}) G_{\alpha \beta}^{(l)}(\boldsymbol{q}, \omega)=\left\langle\left\langle S_{a}^{+} ;\left(S_{\beta}^{Z}\right)^{l} S_{\beta}^{-}\right\rangle\right\rangle_{\boldsymbol{q}}^{\omega}(\alpha \beta=A A, B A, B B, A B)$, $l$ is a positive integer number: $l \leq 2 S, \boldsymbol{q}\left(q_{x}, q_{y}\right)$ is a wave vector). The remaining higher order GFs in the equations of motion are decoupled by the Tyablikov approximation and by the generalized Callen decoupling [2]. We finally obtain the equations of motion in the following matrix form:

$$
P\left(\begin{array}{cc}
G_{A A}^{(l)}(\boldsymbol{q}, \omega) & G_{A B}^{(l)}(\boldsymbol{q}, \omega) \\
G_{B A}^{(l)}(\boldsymbol{q}, \omega) & G_{B B}^{(l)}(\boldsymbol{q}, \omega)
\end{array}\right)=\left(\begin{array}{cc}
Z_{A}^{(l)} & 0 \\
0 & Z_{B}^{(l)}
\end{array}\right) \text {, where } P=\left(\begin{array}{cc}
\omega-J_{B 0}^{A \boldsymbol{q}}+h & m_{A} J_{\boldsymbol{q}} \\
m_{B} J_{\boldsymbol{q}} & \omega-J_{A 0}^{B \boldsymbol{q}}+h
\end{array}\right) .
$$

We define for the square lattice the following abbreviations:

$$
\begin{aligned}
& J_{B 0}^{A \boldsymbol{q}}=J_{B 0}+J_{A \boldsymbol{q}}, \quad J_{A 0}^{B \boldsymbol{q}}=J_{A 0}+J_{B \boldsymbol{q}}, J_{\alpha 0}=4\left\langle S_{\alpha}^{Z}\right\rangle(J+D), Z_{\alpha}^{(l)}=\left\langle\left[S_{\alpha}^{Z},\left(S_{\alpha}^{Z}\right)^{l} S_{\alpha}^{-}\right]\right\rangle, J_{\boldsymbol{q}}=4 J \cos \left(q_{x} / 2\right) \cos \left(q_{y} / 2\right), \\
& J_{\alpha} \boldsymbol{q}=J_{\alpha}\left\langle S_{\alpha}^{Z}\right\rangle\left(4-2\left[\cos q_{x}+\cos q_{y}\right]\right), \tan \theta_{\alpha}=\frac{h^{x}-K_{2}\left\langle S_{\alpha}^{Z}\right\rangle \sin ^{3} \theta_{\alpha} Q^{\left(S_{\alpha}\right)}}{2 K_{2}\left\langle S_{\alpha}^{Z}\right\rangle \cos \theta_{\alpha}\left[1-\sin ^{2}\left(\theta_{\alpha}\right) / 2\right] Q_{\alpha}^{\left(S_{\alpha}\right)}} .
\end{aligned}
$$

GFs have simple poles $\omega^{ \pm}$, which can be obtained by solving the equation $|P|=0$. Equations (3) can be solved by finding the inverse matrix $P^{-1}$. We obtain for $G_{A A}^{(l)}(\boldsymbol{q}, \omega)$ the following form:

$$
G_{A A}^{(l)}(\boldsymbol{q}, \omega)=\frac{Z_{A}^{(l)}}{\omega^{-}-\omega^{+}}\left(\frac{J_{A 0}^{B \boldsymbol{q}}-h-\omega^{+}}{\omega-\omega^{+}}-\frac{J_{A 0}^{B \boldsymbol{q}}-h-\omega^{-}}{\omega-\omega^{-}}\right) .
$$

After using the spectral theorem, we finally obtain the correlation function $C_{A}^{(l)}=\left\langle\left(S_{A}^{Z}\right)^{l} S_{A}^{-} S_{A}^{+}\right\rangle$for the sublattice $A$ 
as

$$
\left\langle\left(S_{A}^{Z}\right)^{l} S_{A}^{-} S_{A}^{+}\right\rangle=Z_{A}^{(l)} \Phi_{A}, \quad(5) \quad \text { where } \quad \Phi_{A}=\frac{1}{\pi^{2}} \int_{0}^{\pi} \int_{0}^{\pi} \frac{d q_{x} d q_{y}}{\omega^{-}-\omega^{+}}\left(\frac{J_{A 0}^{B \boldsymbol{q}}-h-\omega^{+}}{e^{\beta \omega^{+}}-1}-\frac{J_{A 0}^{B \boldsymbol{q}}-h-\omega^{-}}{e^{\beta \omega^{-}}-1}\right) .
$$

The integral in (6) goes over $q_{x}$ and $q_{y}$ in the first Brillouin zone which corresponds to a square lattice.

The Green function $G_{B B}^{(l)}(\boldsymbol{q}, \omega)$ and the function $\Phi_{B}$ can be obtained from (4) and (6) by substitution of indices $A \leftrightarrow B$. From (5) we obtain for $S \geq 1$ the system of algebraic equations for calculation of $\left\langle S_{A}^{Z}\right\rangle$ or $\left\langle S_{B}^{Z}\right\rangle$ (after substitution $A \leftrightarrow B)$.

\section{Results and conclusions}

The components of the magnetization per site in the fixed system $(x, y, z)$ can be calculated from the equations $m_{A}^{z} \equiv\left\langle S_{A}^{z}\right\rangle=\left\langle S_{A}^{Z}\right\rangle \cos \theta_{A}, m_{A}^{x} \equiv\left\langle S_{A}^{x}\right\rangle=\left\langle S_{A}^{Z}\right\rangle \sin \theta_{A}$, (the sublattice $A$ ) and $m_{B}^{x} \equiv\left\langle S_{B}^{x}\right\rangle=-\left\langle S_{B}^{Z}\right\rangle \sin \theta_{B}$, $m_{B}^{z} \equiv\left\langle S_{B}^{z}\right\rangle=-\left\langle S_{B}^{Z}\right\rangle \cos \theta_{B}$ (the sublattice $B$ ). The spins for the sublattice $A$ and $B$ are $3 / 2$ and 1 , respectively.

The reorientation temperatures $T_{R \alpha}$ is defined as the temperature at which the longitudinal magnetization $m_{\alpha}^{z}$ vanishes, the transverse magnetization $m_{\alpha}^{x}$ is nonzero and the orientation angle $\left|\theta_{\alpha}\right|=90^{\circ}$. This can be seen in Fig. 1, where we plot the $z$ and $x$ components of the magnetization and the orientation angle $\theta_{\alpha}$ as functions of the reduced temperature $k T / J$ in the sublattices $A$ and $B$.
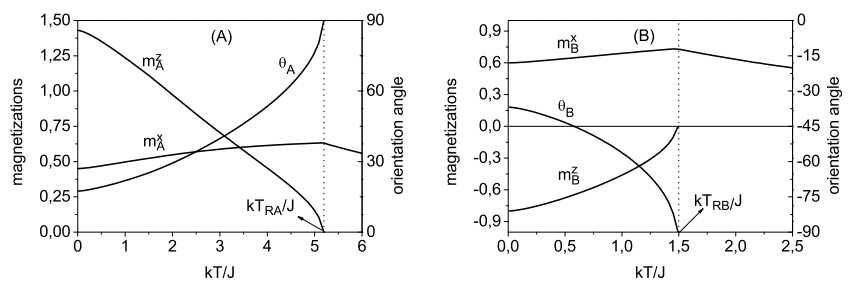

Fig. 1. The components of the magnetization and the orientation angles as functions of the reduced temperature $k T / J$ are shown for the sublattice $A(\mathrm{~A})$ and for the sublattice $B(\mathrm{~B})$ for the parameters: $J=1.0, J_{A} / J=$ $J_{B} / J=0.001, D / J=K_{2} / J=0.01, h^{x} / J=0.006$.

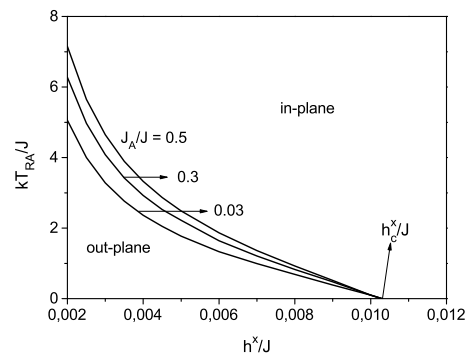

Fig. 2. The reduced reorientation temperature $k T_{R A} / J$ is shown as a function of the reduced transverse magnetic field $h^{x} / J$ for the parameters $J=1.0$, $J_{B} / J=0.1, K_{2} / J=D / J=0.005$. The intrasublattice exchange interaction energies $J_{A} / J$ are different.

The difference between the Neel's theory and the present Green function method is that Neel's theory predicts a temperature $T_{N}$ at which both the sublattice
$A$ and $B$ magnetizations become equal to zero. The magnons in our considered system are a complicated mixture of the transversal fluctuations of the sublattice $A$ and $B$ spins. As a result, the magnon fluctuations supress in different ways the magnetic orders of the $A$ and $B$ sublattices and one obtains two magnetic orders [6]. Each of these orders has a different reorientation temperature.

In Fig. 2, the reduced reorientation temperature $k T_{R A} / J$ of the whole system as a function of the reduced transverse magnetic field $h^{x} / J$ is presented for different reduced intra-sublattice exchange interaction energies $J_{A} / J$. For $h^{x} / J>h_{C}^{x} / J$ the direction of the $z$ component of magnetization of the whole system is in the plane of the monolayer and the magnetic reorientation does not appear. On the other hand, the magnetic reorientation can be observed for $h^{x} / J<h_{C}^{x} / J$ by increasing the temperature. The critical $h_{C}^{x} / J$ value does not depend on the sublattice interaction $J_{A}$.

In [6], the two-sublattice ferrimagnet with different spins and different intra-sublattice ferromagnetic exchange interaction was considered within the modified spin-wave theory and two magnetic orders were obtained. Unlike the model in [6], we have included in the Hamiltonian the single-ion anisotropy and the transverse magnetic field to consider magnetic reorientation within the Green function theory. We obtained two magnetic orders; each of these orders has different reorientation temperatures (Fig. 1). Next, we determined the boundary between two states of the whole ferrimagnetic system with different orientations of the $z$-component of the magnetization (in-plane and out of plane), see Fig. 2.

\section{Acknowledgments}

I wish to thank the JINR Directorate for kind hospitality. This work was supported by JINR Grant, 2013, No 114.

\section{References}

[1] P. Fröbrich, P.J. Kuntz, Physics Reports. 432, 223 (2006).

[2] S. Schwieger, J. Kienert, W. Nolting, Phys. Rev. B 71, 024428 (2005).

[3] V. Ilkovič, Phys. Status Solidi B 246, 2366 (2009).

[4] V. Ilkovič, J. Kecer, Phys. Status Solidi B 249, 181 (2012).

[5] H. Zengh and D.L. Lin, Phys. Rev. B 37, 9615 (1988).

[6] N. Karchev, J. Phys.: Condens. Matter 20, 325219 (2008). 